\title{
SOME TRIPLE TRIGONOMETRICAL SERIES
}

\author{
By C. J. TRANTER
}

(Received 15 April, 1968; revised 18 October, 1968)

1. Introduction. This paper considers the determination of the coefficients in two sets of triple trigonometrical series and shows that these can be obtained in closed form. The series considered are special cases of some triple series in Jacobi polynomials studied by $\mathrm{K}$. N. Srivastava [1]. Srivastava, however, shows that the problem for the more general series can be reduced to the solution of a Fredholm integral equation of the second kind and he does not discuss special cases which may lead to closed form solutions.

By employing an idea due to Williams [2], I reduce the problem to one in triple integral equations and I first discuss the determination of the coefficients $A_{n}$ in the triple series

$$
\begin{array}{ll}
\sum_{n=1}^{\infty} A_{n} \sin n x=0 & (0 \leqq x<\alpha \text { and } \beta<x \leqq \pi), \\
\sum_{n=1}^{\infty} n A_{n} \sin n x=f(x) & (\alpha<x<\beta),
\end{array}
$$

where $f(x)$ is a prescribed function of $x$. I find that these coefficients are given by

$$
A_{n}=2 \int_{0}^{\infty} A(t) J_{2 n}(t) d t,
$$

where the function $A(t)$ satisfies the triple integral equations

$$
\begin{array}{ll}
\int_{0}^{\infty} A(t) \sin u t d t=0 & \left(0 \leqq u<\sin \frac{1}{2} \alpha \text { and } \sin \frac{1}{2} \beta<u<\infty\right), \\
\int_{0}^{\infty} t A(t) \sin u t d t=\frac{2}{\sqrt{\left(1-u^{2}\right)}} f\left(2 \sin ^{-1} u\right) & \left(\sin \frac{1}{2} \alpha<u<\sin \frac{1}{2} \beta\right) .
\end{array}
$$

As I have already given a method [3] for finding $A(t)$ from these triple integral equations, the determination of the coefficients $A_{n}$ in the triple trigonometrical series (1) and (2) is theoretically possible.

In boundary value problems in which the triple series (1) and (2) occur, interest often lies more in the determination of the function $V(x)$ given by

$$
V(x)=\sum_{n=1}^{\infty} A_{n} \sin n x \quad(\alpha<x<\beta)
$$

than in that of the actual coefficients $A_{n}$. It is therefore worth noting that this function can be found from the relation

$$
V\left(2 \sin ^{-1} u\right)=\int_{0}^{\infty} A(t) \sin u t d t \quad\left(\sin \frac{1}{2} \alpha<u<\sin \frac{1}{2} \beta\right),
$$


and that, in the analysis of the triple integral equations (4) and (5), the integral in equation (7) is rather more easily found than the function $A(t)$ itself.

The analysis which leads to the above results is given in Section 2 below and this is followed in Section 3 by a worked example. The final section of this note gives the corresponding results when the sines in series (1) and (2) are replaced by cosines and when $n$ is replaced by $n-\frac{1}{2}$.

2. The analysis. From equations (1) and (6),

$$
A_{n}=\frac{2}{\pi} \int_{\alpha}^{\beta} V(x) \sin n x d x=\frac{4}{\pi} \int_{\sin \frac{1}{2} \alpha}^{\sin \frac{1}{2} \beta} V\left(2 \sin ^{-1} u\right) \frac{\sin \left(2 n \sin ^{-1} u\right)}{\sqrt{\left(1-u^{2}\right)}} d u
$$

where $u=\sin \frac{1}{2} x$. Now [4, p. 405]

$$
\int_{0}^{\infty} J_{2 n}(t) \sin u t d t=\frac{\sin \left(2 n \sin ^{-1} u\right)}{\sqrt{ }\left(1-u^{2}\right)} \quad(0<u<1),
$$

so that the relation (3) is established by writing

$$
A(t)=\frac{2}{\pi} \int_{\sin \frac{1}{\ddagger} \alpha}^{\sin \frac{1}{\ddagger} \beta} V\left(2 \sin ^{-1} u\right) \sin t u d u .
$$

Equation (8) implies the validity of relations (4) and (7) so that it only remains to establish equation (5) from equation (2).

This can be done by using Jacobi's expansion in series of Bessel coefficients; this is $[4$, p. 22]

$$
\cos \left(t \sin \frac{1}{2} x\right)=J_{0}(t)+2 \sum_{n=1}^{\infty} J_{2 n}(t) \cos n x
$$

and differentiation with respect to $x$ gives

$$
\frac{1}{2} t \cos \frac{1}{2} x \sin \left(t \sin \frac{1}{2} x\right)=2 \sum_{n=1}^{\infty} n J_{2 n}(t) \sin n x .
$$

Hence, from equations (2), (3) and (9), when $\alpha<x<\beta$,

$$
\begin{aligned}
f(x) & =\sum_{n=1}^{\infty} n A_{n} \sin n x=2 \int_{0}^{\infty} A(t) \sum_{n=1}^{\infty} n J_{2 n}(t) \sin n x d t \\
& =\int_{0}^{\infty} \frac{1}{2} t A(t) \cos \frac{1}{2} x \sin \left(t \sin \frac{1}{2} x\right) d t,
\end{aligned}
$$

and this is equation (5) when $\sin \frac{1}{2} x$ is replaced by $u$. 
3. An example. I take $f(x)=\sin x$, so that the triple integral equations (4) and (5) are

$$
\left.\begin{array}{ll}
\int_{0}^{\infty} A(t) \sin u t d t=0 & \left(0 \leqq u<\sin \frac{1}{2} \alpha \text { and } \sin \frac{1}{2} \beta<u<\infty\right), \\
\int_{0}^{\infty} t A(t) \sin u t d t=4 u & \left(\sin \frac{1}{2} \alpha<u<\sin \frac{1}{2} \beta\right) .
\end{array}\right\}
$$

These equations can be solved by writing

$$
A(t)=t^{-1} \sum_{r=1}^{\infty}(-1)^{r-1} c_{r} J_{2 r}\left(t \sin \frac{1}{2} \beta\right)
$$

and using the results $[4, \mathrm{p} .405]$

and

$$
\int_{0}^{\infty} J_{2 r}\left(t \sin \frac{1}{2} \beta\right) \sin u t \frac{d t}{t}=\left\{\begin{array}{cl}
\frac{1}{2 r} \sin \left\{2 r \sin ^{-1}\left(u \operatorname{cosec} \frac{1}{2} \beta\right)\right\} & \left(u<\sin \frac{1}{2} \beta\right), \\
0 & \left(u>\sin \frac{1}{2} \beta\right),
\end{array}\right.
$$

$$
\int_{0}^{\infty} J_{2 r}\left(t \sin \frac{1}{2} \beta\right) \sin u t d t=\frac{\sin \left\{2 r \sin ^{-1}\left(u \operatorname{cosec} \frac{1}{2} \beta\right)\right\}}{\sqrt{\left(\sin ^{2} \frac{1}{2} \beta-u^{2}\right)}} \quad\left(u<\sin \frac{1}{2} \beta\right) .
$$

Thus we find that the form chosen in (11) for $A(t)$ automatically gives $\int_{0}^{\infty} A(t) \sin u t d t=0$ when $\sin \frac{1}{2} \beta<u<\infty$ and that the other two of equations (10) require that the coefficients $c_{r}$ should satisfy the dual relations

$$
\left.\begin{array}{ll}
\sum_{r=1}^{\infty} c_{r} \sin r \theta=2 \sin ^{2} \frac{1}{2} \beta \sin \theta & (0 \leqq \theta<\gamma), \\
\sum_{r=1}^{\infty} r^{-1} c_{r} \sin r \theta=0 & (\gamma<\theta \leqq \pi),
\end{array}\right\}
$$

where

$$
u=\cos \frac{1}{2} \theta \sin \frac{1}{2} \beta, \quad \cos \frac{1}{2} \gamma=\sin \frac{1}{2} \alpha \operatorname{cosec} \frac{1}{2} \beta .
$$

It is also found that equation (7) becomes

$$
V\left(2 \sin ^{-1} u\right)=\int_{0}^{\infty} A(t) \sin u t d t=\frac{1}{2} \sum_{r=1}^{\infty} r^{-1} c_{r} \sin r \theta \quad(0 \leqq \theta<\gamma)
$$

Using the method given by Sneddon [5, p. 152] for the solution of the dual series (14), we find that, when $0 \leqq \theta<\gamma$,

$$
\sum_{r=1}^{\infty} r^{-1} c_{r} \sin r \theta=\sin \frac{1}{2} \theta \int_{\theta}^{\gamma} \frac{h(t) d t}{\sqrt{(\cos \theta-\cos t)}}
$$


where

$$
h(t)=\frac{2}{\pi} \cot \frac{1}{2} t \int_{0}^{t} \frac{2 \sin ^{2} \frac{1}{2} \beta \sin \theta \sin \frac{1}{2} \theta}{\sqrt{(\cos \theta-\cos t)}} d \theta .
$$

Since $2 \sin \theta \sin \frac{1}{2} \theta=\cos \frac{1}{2} \theta-\cos \frac{3}{2} \theta$, use of Mehler's integral for $P_{n}(\cos t)$ shows that

$$
h(t)=\frac{2}{\pi} \cot \frac{1}{2} t \sin ^{2} \frac{1}{2} \beta \frac{\pi}{\sqrt{2}}\left\{P_{0}(\cos t)-P_{1}(\cos t)\right\}=\sqrt{ } 2 \sin ^{2} \frac{1}{2} \beta \sin t .
$$

Hence, when $0 \leqq \theta<\gamma$,

$$
\sum_{r=1}^{\infty} r^{-1} c_{r} \sin r \theta=\sqrt{ } 2 \sin ^{2} \frac{1}{2} \beta \sin \frac{1}{2} \theta \int_{\theta}^{\gamma} \frac{\sin t d t}{\sqrt{(\cos \theta-\cos t)}}=2 \sqrt{2} \sin ^{2} \frac{1}{2} \beta \sin \frac{1}{2} \theta \sqrt{ }(\cos \theta-\cos \gamma) .
$$

Using equations (15) and (16), we find that, when $\sin \frac{1}{2} \alpha<u<\sin \frac{1}{2} \beta$,

$$
V\left(2 \sin ^{-1} u\right)=2\left(u^{2}-\sin ^{2} \frac{1}{2} \alpha\right)^{\frac{1}{2}}\left(\sin ^{2} \frac{1}{2} \beta-u^{2}\right)^{\frac{1}{2}} .
$$

Hence, when $\alpha<x<\beta$,

$$
V(x)=2\left(\sin ^{2} \frac{1}{2} x-\sin ^{2} \frac{1}{2} \alpha\right)^{\frac{1}{2}}\left(\sin ^{2} \frac{1}{2} \beta-\sin ^{2} \frac{1}{2} x\right)^{\frac{1}{2}}=(\cos \alpha-\cos x)^{\frac{1}{2}}(\cos x-\cos \beta)^{\frac{1}{2}}
$$

and equations (1) and (6) then give

$$
A_{n}=\frac{2}{\pi} \int_{\alpha}^{\beta}(\cos \alpha-\cos x)^{\frac{1}{2}}(\cos x-\cos \beta)^{\frac{1}{2}} \sin n x d x .
$$

4. Triple cosine series. The coefficients $B_{n}$ in the triple cosine series

$$
\begin{aligned}
\sum_{n=1}^{\infty} B_{n} \cos \left(n-\frac{1}{2}\right) x & =0 \quad(0 \leqq x<\alpha \text { and } \beta<x \leqq \pi), \\
\sum_{n=1}^{\infty}\left(n-\frac{1}{2}\right) B_{n} \cos \left(n-\frac{1}{2}\right) x & =f(x) \quad(\alpha<x<\beta),
\end{aligned}
$$

can be found by analysis similar to that given in Section 2 above. The result is

$$
B_{n}=2 \int_{0}^{\infty} B(t) J_{2 n-1}(t) d t
$$

where $B(t)$ satisfies the triple integral equations

$$
\begin{aligned}
& \int_{0}^{\infty} B(t) \cos u t d t=0 \quad\left(0 \leqq u<\sin \frac{1}{2} \alpha \quad \text { and } \quad \sin \frac{1}{2} \beta<u<\infty\right) \\
& \int_{0}^{\infty} t B(t) \cos u t d t=\frac{2}{\sqrt{\left(1-u^{2}\right)}} f\left(2 \sin ^{-1} u\right) \quad\left(\sin \frac{1}{2} \alpha<u<\sin \frac{1}{2} \beta\right) .
\end{aligned}
$$


It can also be shown that, if

$$
W(x)=\sum_{n=1}^{\infty} B_{n} \cos \left(n-\frac{1}{2}\right) x \quad(\alpha<x<\beta),
$$

then

$$
W\left(2 \sin ^{-1} u\right)=\int_{0}^{\infty} B(t) \cos u t d t \quad\left(\sin \frac{1}{2} \alpha<u<\sin \frac{1}{2} \beta\right)
$$

\section{REFERENCES}

1. K. N. Srivastava, On triple integral series equations involving series of Jacobi polynomials, Proc. Edinburgh Math. Soc. 15 (1967), 221-231.

2. W. E. Williams, Note on the reduction of dual and triple series equations to dual and triple integral equations, Proc. Cambridge Philos. Soc. 59 (1963), 731-734.

3. C. J. Tranter, Some triple integral equations, Proc. Glasgow Math. Assoc. 4 (1960), 200-203.

4. G. N. Watson, Theory of Bessel functions (Cambridge, 1944).

5. I. N. Sneddon, Mixed boundary value problems in potential theory (New York, 1966).

Royal Military College of Science SHRIVENHAM 